\title{
Does long-term oxygen therapy reduce hospitalisation in hypoxaemic chronic obstructive pulmonary disease?
}

\author{
T.J. Ringbaek*, K. Viskum*, P. Lange*
}

Does long-term oxygen therapy reduce hospitalisation in hypoxaemic chronic obstructive pulmonary disease? T.J. Ringbaek, K. Viskum, P. Lange. C) ERS Journals Ltd 2002.

ABSTRACT: The aim of this study was to determine whether long-term oxygen therapy (LTOT) reduces hospitalisation in hypoxaemic patients with chronic obstructive pulmonary disease (COPD). The circumstances of initiation of LTOT (start during hospitalisation versus start as an outpatient) and compliance with oxygen were also investigated (continuous oxygen therapy (COT) $\geqslant 15 \mathrm{~h}$ daily versus noncontinuous oxygen therapy (NCOT) $<15$ h daily).

A total 246 COPD patients were studied, with each patient acting as their own control. Patients were divided into four groups: 125 patients on COT who started LTOT in conjunction with hospitalisation, 37 patients on COT who started LTOT as outpatients, 58 patients on NCOT who started LTOT in conjunction with hospitalisation and 26 patients on NCOT who started LTOT as outpatients. Admission rates, days spent in hospital and number of patients with at least one hospitalisation (ever hospitalised) were compared in two periods of $\mathbf{1 0}$ months before and after initiation of LTOT.

Overall during the LTOT period, in comparison with the preoxygen period, the admission rates, hospital days and "ever hospitalised" were reduced by $23.8 \%, 43.5 \%$ and $31.2 \%$, respectively. Among patients who started LTOT as outpatients, a tendency towards a higher effect in the compliant group was observed.

This study shows that in hypoxaemic chronic obstructive pulmonary disease patients, long-term oxygen therapy is associated with a reduction in hospitalisation. Eur Respir J 2002; 20: 38-42.
*Dept of Respiratory Medicine, Hvidovre Hospital, University Hospital of Copenhagen, Hvidovre and ${ }^{\#}$ Dept of Respiratory Medicine, Gentofte University, University Hospital of Copenhagen, Gentofte, Denmark.

Correspondence: T.J. Ringbaek

Krogebakke 2 B

DK-3140 Aalsgaarde

Denmark

Fax: 4536323716

E-mail: ringbaek@dadlnet.dk

Keywords: Home care hospitalisation

obstructive lung diseases

oxygen inhalation therapy

Received: October 22001

Accepted after revision: February 6 2002

This study was supported by The Danish Lung Association.
Long-term oxygen therapy (LTOT) is a widely used treatment that improves survival in chronic obstructive lung disease (COPD) with severe hypoxaemia [1-3]. Since the cost of home oxygen therapy is high, demonstration of any economical advantage from a reduction in hospitalisation related to use of LTOT is important [4]. An early study from the 1970s and two recent studies, all including a small number of patients acting as their own control, have indicated that LTOT decreases hospitalisations [5-7]. However, a Medical Research Council (MRC) study, which had a randomised-control group, failed to confirm this advantage of LTOT [1]. Today, it is considered unethical to undertake placebo-controlled studies in COPD patients with chronic hypoxaemia. Conversely, studies with patients acting as their own control may be biased by the fact that frequent hospitalisations and the decision to prescribe LTOT are interrelated; physicians may be more likely to initiate LTOT in patients with frequent hospitalisations rather than in patients with a stable condition. A reduction in hospitalisations after initiation of LTOT could therefore simply reflect a "regression to the mean phenomenon", a bias that has not been focused on in previous studies [5-7]. Taking this into account, the effect of LTOT on hospitalisations, in a larger study with patients acting as their own control, was investigated. In addition, given that not all patients use oxygen for the recommended number of hours (at least 15 daily), the authors investigated whether compliance with hours spent on oxygen had an impact on hospitalisation.

\section{Methods}

Information from the Danish Oxygen Register, which was established in November 1994 and covers $98 \%$ of the Danish population, was used. From the patients' hospital files and general practitioner files, information on diagnosis, medical treatment, smoking habits, arterial blood gas tensions, body mass index (BMI), and forced expiratory volume in one second (FEV1) were obtained. The best arterial blood test obtained within the period 1 month before to 3 months after initiation of LTOT was registered. Evidence of cor pulmonale was assessed from the latest electrocardiogram in the study period. Within 1 month after initiation of LTOT, patients received a questionnaire by mail on the use of oxygen (number of hours daily) and outdoor activity (yes or no). The National Board of Health provided information on 
hospital admissions and all contacts to outpatient clinics. Data on delivered oxygen systems were obtained from the oxygen suppliers.

The study comprised 246 COPD hypoxaemic patients who were divided into four groups depending on their use of oxygen (continuous oxygen therapy (COT) (patients using oxygen $\geqslant 15 \mathrm{~h}$ daily) versus noncontinuous oxygen therapy (NCOT) (patients using $<15 \mathrm{~h}$ daily)) and the circumstances of initiation of oxygen therapy (start during hospitalisation versus start as an outpatient) (fig. 1). Subclassification according to circumstances of initiation of oxygen therapy was owing to a hypothesis that an effect of oxygen therapy in patients who started home oxygen therapy as outpatients was less likely to be derived from a "regression to the mean phenomenon". Patients with NCOT were either prescribed oxygen $<15 \mathrm{~h}$ daily or were prescribed COT but used it insufficiently (poor compliance). The two groups of patients with NCOT did not differ from each other regarding days spent in hospital and number of

a)

Patients started LTOT 01/11/94-31/07/95: $n=1,591$
Non-COPD disease: $n=767$
Diagnosis unknown: $n=2$
D total of 822 had COPD $(51.7 \%)$

$\downarrow$ Data on hypoxaemic status: $n=169$ (20.6\%)

A total of 653 with COPD and data on hypoxaemic status

$$
\begin{gathered}
\text { No data on usage of oxygen: } n=309(47.3 \%) \\
\text { Nonhypoxaemic patients: } n=98(15.0 \%) \\
\text { Patients encompassing four groups: } n=246
\end{gathered}
$$

b)

Usage of oxygen 15-24 h.day-1 (COT) $\mathrm{n}=162$ A total 125 started oxygen therapy in connections with hospitalisation

A total 37 started oxygen therapy as outpatients

Usage of oxygen $<15$ h.day ${ }^{-1}$ (NOT)

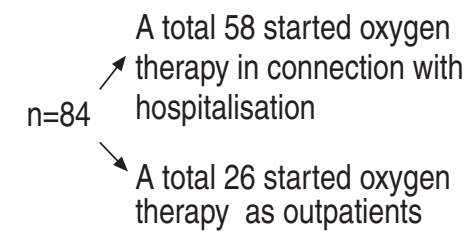

Fig. 1.-a) Flow chart of patients on long-term oxygen therapy and $b$ ) the division of the four groups. COPD: chronic obstructive pulmonary disease; COT: continuous oxygen therapy; NCOT: noncontinuous oxygen therapy; hypoxemic status: oxygen tension in arterial blood $<7.3 \mathrm{kPa}(50 \mathrm{mmHg})$ or $7.3-8.0 \mathrm{kPa}(50-60 \mathrm{mmHg})$ together with cor pulmonale according to electrocardiogram, while resting and on room air. admissions before and after the start of home oxygen therapy. A previous Danish study has shown a high correlation between the actual use of oxygen and the reported use of oxygen [8]. Except for usage of oxygen and prescribed mobile oxygen, small differences were found between the COT and NCOT patients (table 1). All patients had a clinical diagnosis of COPD, which in most cases was confirmed by a lung function test. Only the best spirometry value obtained during the study period was included in this study. Hospital admission rates and numbers of days in hospital were compared over two 10-month periods, before (preoxygen period) and after (oxygen period) initiation of LTOT therapy. If oxygen therapy was stopped within 10 months ( $n=49$ patients), the preoxygen period was reduced correspondingly, omitting days from the beginning of the period. The average observation time before and after LTOT was 283 days, median time 304 days (range 46-304 days). Although reassessment is recommended 1-3 months after the start of treatment, only 50 of $147(34 \%)$ patients who started LTOT immediately after a hospitalisation and received oxygen for at least 3 months had hypoxaemic status certified 1-3 months after start of treatment.

None of the patients had been treated with home mechanical ventilation, had a lung transplantation, had undergone lung-volume reduction surgery or a pulmonary rehabilitation programme during the study period.

The Danish entry criteria for LTOT are in accordance with international guidelines, and re-assessment

Table 1.-Characteristics of 246 chronic obstructive pulmonary disease patients according to usage of oxygen

\begin{tabular}{|c|c|c|c|}
\hline & $\begin{array}{l}\text { Subjects } \\
\mathrm{n}\end{array}$ & $\mathrm{COT}^{\S}$ & $\mathrm{NCOT}^{f}$ \\
\hline Age yrs & & $68.4 \pm 8.7$ & $71.6 \pm 7.4$ \\
\hline Sex female $\%$ & & 56.8 & 48.8 \\
\hline Current smoker \% & 245 & 16.0 & 20.5 \\
\hline Body mass index $\mathrm{kg} \cdot \mathrm{m}^{-2}$ & 228 & $22.0 \pm 6.0$ & $22.0 \pm 4.6$ \\
\hline Had outdoor activity \% & 242 & 66.0 & 72.3 \\
\hline Systemic corticosteroid \% & 245 & 50.9 & 44.0 \\
\hline FEV $1 \%$ pred value & 114 & $29.6 \pm 10.7$ & $30.2 \pm 10.9$ \\
\hline $\begin{array}{l}\text { Prescribed oxygen } \\
\text { flow } \mathrm{L} \cdot \mathrm{min}^{-1}\end{array}$ & & $1.3 \pm 0.6$ & $1.3 \pm 0.7$ \\
\hline 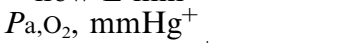 & & $6.38 \pm 0.7$ & $6.36 \pm 0.9$ \\
\hline $\mathrm{Pa}, \mathrm{CO}_{2}, \mathrm{mmHg}^{+}$ & & $6.78 \pm 1.2$ & $6.49 \pm 1.4$ \\
\hline $\begin{array}{l}\text { Time spent with oxygen } \\
\text { according to the } \\
\text { patient } h\end{array}$ & & $18.5 \pm 3.4$ & $8.2 \pm 4.8$ \\
\hline Liquid oxygen $\%$ & 245 & 9.3 & 4.8 \\
\hline Concentrator $\%$ & 245 & 79.6 & 79.8 \\
\hline Mobile oxygen \% & 245 & 49.7 & 29.8 \\
\hline
\end{tabular}
(continuous oxygen therapy ${ }^{\#}$ (COT) versus noncontinuous oxygen therapy" (NCOT))

Data are presented as mean \pm SD unless otherwise stated. FEV1: forced expiratory volume in one second; $P \mathrm{a}_{2} \mathrm{O}_{2}$ : oxygen tension in arterial blood, COT $=48.0$ (5.3) $\mathrm{mmHg}$, $\mathrm{NCOT}=47.8$ (7.1) $\mathrm{mmHg} ; \mathrm{Pa}_{2} \mathrm{CO}_{2}$ : carbon dioxide tension in arterial blood, COT=51.0 (9.0) $\mathrm{mmHg}, \mathrm{NCOT}=48.8(10.5)$ mmHg. ${ }^{\S}$ : $\mathrm{n}=162 ;{ }^{f}: \mathrm{n}=84$; ${ }^{\#}: 15-24 \mathrm{~h} \cdot$ day $^{-1}$; ${ }^{\uparrow}:<15 \mathrm{~h} \cdot$ day $^{-1}$; ${ }^{+}: 20$ patients had only blood gases measured while on supplemental oxygen. 
Table 2. - Days spent in hospital and admission rates before and after starting long-term oxygen therapy (LTOT) in two groups of hypoxaemic chronic obstructive pulmonary disease patients depending on compliance with hours spent with oxygen

\begin{tabular}{|c|c|c|c|c|c|}
\hline Variable & Subjects $n$ & Before LTOT & After LTOT & Relative change ${ }^{\#} \%$ & p-value \\
\hline All & 256 & & & & \\
\hline Days & & $23.7 \pm 24.5$ & $13.4 \pm 22.7$ & 43.5 & $<0.001$ \\
\hline Admission & & $2.1 \pm 1.9$ & $1.6 \pm 2.2$ & 23.8 & $<0.001$ \\
\hline Ever admitted \% & & 92.7 & 63.8 & 31.2 & $<0.001$ \\
\hline COT & 162 & & & & \\
\hline Days & & $23.1 \pm 23.9$ & $14.2 \pm 25.7$ & 38.5 & $<0.001$ \\
\hline Admission & & $2.2 \pm 1.9$ & $1.6 \pm 2.0$ & 27.3 & $<0.001$ \\
\hline Ever admitted \% & & 94 & 63 & 33.0 & $<0.001$ \\
\hline NCOT & 84 & & & & \\
\hline Days & & $24.9 \pm 25.7$ & $11.9 \pm 15.3$ & 52.6 & $<0.001$ \\
\hline Admission & & $2.0 \pm 1.7$ & $1.7 \pm 2.6$ & 15.0 & 0.28 \\
\hline Ever admitted \% & & 89 & 65 & 27.0 & 0.001 \\
\hline
\end{tabular}

Data are presented as mean \pm SD unless otherwise stated. COT: continuous oxygen therapy $\left(15-24 \mathrm{~h} \cdot \mathrm{day}^{-1}\right)$; NCOT: noncontinuous oxygen therapy $\left(<15 \mathrm{~h} \cdot \mathrm{day}^{-1}\right)$. ${ }^{\#}$ : (preoxygen period-oxygen period)/preoxygen period.

is recommended four times annually. The regional ethical committees and the Data Inspection Board approved the study.

\section{Statistics}

The Wilcoxon test was used to compare admission rates and days in hospital in pre- and postoxygen periods. The Sign test was used to compare "ever hospitalised". The Mann-Whitney U-test was used to compare the effect of home oxygen therapy on spent days in hospital. Two-sided tests were employed. A $p<0.05$ was considered significant.

\section{Results}

Among the 246 patients, LTOT reduced hospital days, admission rates and number of patients with at least one hospitalisation by $43.5 \%, 23.8 \%$ and $31.2 \%$, respectively (table 2 ).

Most of the 162 COT patients $(77.2 \%)$ started oxygen therapy immediately after a hospitalisation, and only $46(14.2 \%)$ had not been hospitalised during a 2-month period prior to initiation of oxygen therapy. In comparison with the preoxygen period, hospitalisation and days spent in hospital were reduced by $\sim 30-40 \%$ during the oxygen period (table 2). When the effect of COT on days spent in the hospital was investigated in patients who started oxygen therapy as an outpatient, a similar reduction was found in days spent in hospital and number of patients with a hospitalisation, however the admission rates were not reduced (table 3 ).

Compared to patients with COT, patients with NCOT had a similar reduction of hospitalisation (table 2). Patients who started COT as outpatients seemed to benefit more from oxygen therapy compared to those with NCOT, but this difference did not reach statistical significance (table 3).

\section{Discussion}

The first months of LTOT were found to be associated with a significant reduction of days spent in hospital, as compared to the preoxygen period. This reduction is comparable with findings of previous studies where reductions of $40-50 \%$ were observed [5-7]. With regard to severity of COPD, the patients in this study seem to be similar to those studied previously as the oxygen tension in arterial blood

Table 3.-Days spent in hospital and admission rates before and after starting long-term oxygen therapy (LTOT) in two hypoxaemic groups of chronic obstructive pulmonary disease patients, who started oxygen therapy in the outpatient clinic, depending on compliance with hours spent with oxygen

\begin{tabular}{|c|c|c|c|c|c|}
\hline Variable & Subjects $n$ & Before LTOT & After LTOT & Relative change ${ }^{\#} \%$ & $\mathrm{p}$-value \\
\hline $\mathrm{COT}$ & 37 & & & & \\
\hline Days & & $13.8 \pm 19.0$ & $8.4 \pm 11.9$ & 39.1 & 0.16 \\
\hline Admission & & $1.2 \pm 1.0$ & $1.2 \pm 1.4$ & -8.3 & 0.84 \\
\hline Ever admitted \% & & 76 & 57 & 25.0 & 0.17 \\
\hline NCOT & 26 & & & & \\
\hline Days & & $12.2 \pm 19.6$ & $9.2 \pm 9.9$ & 23.8 & 0.46 \\
\hline Admission & & $1.1 \pm 1.6$ & $1.1 \pm 1.1$ & 0 & 1.0 \\
\hline Ever admitted \% & & 65 & 62 & 4.6 & 1.0 \\
\hline
\end{tabular}

Data are presented as mean (SD) unless otherwise stated. COT: continuous oxygen therapy $\left(15-24 \mathrm{~h} \cdot \mathrm{day}^{-1}\right)$; $\mathrm{NCOT}$ noncontinuous oxygen therapy $\left(<15 \mathrm{~h} \cdot \mathrm{day}^{-1}\right)$. ${ }^{\#}$ : (preoxygen period-oxygen period)/preoxygen period. 
$\left(P \mathrm{a}, \mathrm{O}_{2}\right), \mathrm{FEV} 1$ and hospitalisations per patient year were comparable $[6,7,9,10]$. These studies have not provided data on systemic steroid treatment, but the frequency of steroid users in the current study was equal to Swedish COPD patients on LTOT [11].

Home oxygen therapy had a similar effect on patients in both the COT and NCOT groups, this could reflect either a bias from a connection between frequent hospitalisation and initiation of LTOT or a true effect of oxygen therapy. The majority of the COT patients in this study $(77.2 \%)$ started oxygen immediately after a hospitalisation. Similarly, in a large study of all new home oxygen users in the USA in $1992,68 \%$ of the patients had been hospitalised up to 90 days prior to oxygen therapy [12]. Thus, potentially, the observed decrease in hospital days following initiation of COT may reflect a "regression to the mean phenomenon" rather than an effect of oxygen per se. However, when selecting patients who started COT in the outpatient clinic, and are therefore considered more clinically stable, the magnitude of beneficial effect on bed days was still present, although it did not reach statistical significance because of the small numbers. The reduction in admission rates was only present among patients who started COT after hospitalisation. This indicates that the decrease in hospital days, but not the decrease in number of hospitalisations, was related to COT and not to a bias from a connection between frequent hospitalisation and initiation of oxygen therapy. Correspondingly, in a small study of 10 patients on LTOT, a reduction in hospital days was observed, which probably indicated a real effect of LTOT on hospitalisations since the patients did not have increased numbers of hospital days prior to initiation of treatment [6]. These 10 patients, however, may not be representative of the majority of LTOT patients, and a bias from a connection between frequent hospitalisation and initiation of oxygen therapy (even as outpatients) is impossible to role out definitively using a cohort study.

The impact of number of hours on oxygen is less clear. Previously, only the Nocturnal Oxygen Therapy Trial (NOTT) study has focused on hospitalisations where oxygen was used $<15 \mathrm{~h}$ daily [2]. As in the NOTT study, when patients who started home oxygen therapy during a clinically stable condition were investigated, it was found that using oxygen for $\sim 18 \mathrm{~h}$ daily was slightly better than $8-12 \mathrm{~h}$ in terms of reducing hospitalisation in hypoxaemic COPD patients.

According to the study by Silverman et al. [12] the most common causes of hospitalisation in patients on LTOT are heart failure, COPD exacerbations and lung infections. In hypoxaemic patients, any effect from oxygen therapy on hospitalisation could be derived from alleviating attacks of severe desaturations and from stabilising pulmonary haemodynamics $[2,13,14]$. It is reasonable to expect that both NCOT and COT are able to reduce dyspnoea by reducing the effort of breathing and preventing the development of fear and anxiety, thus preventing hospitalisation of some COPD patients in a stable condition even without permanent hypoxaemia [15]. Perhaps
COT and NCOT combined with home care may result in an even more marked reduction of hospitalisation. In support of this hypothesis, introduction of a home-care programme with frequent visits to patients on LTOT has been shown to reduce hospitalisation rates by one-half when compared with both a historical control period and a randomised control group $[10,11]$. In addition, home management has been proven to reduce hospitalisation in COPD patients with exacerbations of COPD [16]. This kind of home management of patients on NCOT or LTOT was not in practise in Denmark at the time of this study.

In the MRC study, the authors speculated that the failure of LTOT to reduce the hospital stay might reflect the close clinical supervision of the patients in both the oxygen treatment and the placebo group [1]. Similarly, one reason for the observed reduction of hospitalisation in the studies using historical comparisons could be intensified visits to the outpatient clinic with adjunctive measures and treatment. However, in the current study only $57(35.2 \%)$ of the COT patients were seen in the outpatient clinic within the first 6 months on oxygen therapy. Another explanation for the failure of LTOT to reduce hospitalisation when compared with placebo, could be a substantial effect of placebo. Several studies have found that placebo (sham oxygen) improves dyspnoea and exercise tolerance $[17,18]$.

Most of the patients in this study started LTOT immediately after hospitalisation, during a clinically unstable condition. Only $50(34 \%)$ of the 147 patients who had LTOT for $>3$ months and were started on LTOT in connection with a hospitalisation had hypoxaemic status certified 1-3 months after initiation of home oxygen therapy. Previous studies have shown that $\sim 40 \%$ of these clinically unstable patients would not meet the criteria for $\mathrm{Pa}_{\mathrm{a}} \mathrm{O}_{2}$ if retested 3 months later [2, 19]. Therefore, it is likely that not all of the patients classified as hypoxaemic had continuous hypoxaemia. However, regarding the effect of oxygen therapy on hospitalisation, unstable patients with certified hypoxaemic status did not differ from those unstable patients without a follow-up.

To conclude, the current study shows that in hypoxaemic chronic obstructive pulmonary disease patients, long-term oxygen therapy is associated with a reduction in days spent in hospital. Furthermore, the beneficial effect of long-term oxygen therapy on hospitalisation seems to reflect an effect of therapy per se and not a "regression to the mean phenomenon".

\section{References}

1. Medical Research Council. Long term domiciliary oxygen therapy in chronic hypoxic cor pulmonale complicating chronic bronchitis and emphysema. Report of a working party. Lancet 1981; 1: 681-686.

2. Nocturnal Oxygen Therapy Trial Group. Continuous or nocturnal oxygen therapy in hypoxemic chronic obstructive lung disease: A clinical trial. Ann Intern Med 1980; 93: 391-398.

3. Gorecha D, Gorzelak K, Sliwinski P, Tobiasz M, 
Zielinski J. Effect of long term oxygen therapy on survival in patients with chronic obstructive pulmonary disease with moderate hypoxaemia. Thorax 1997; 52: 674-679.

4. O'Donohue WJ Jr, Plummer AL. Magnitude of usage and cost of home oxygen therapy in the United States. Chest 1995; 107: 301-302.

5. Crockett AJ, Moss JR, Cranston JM, Alpers JH. The effect of home oxygen therapy on hospital admission rates in chronic obstructive airways disease. Monaldi Arch Chest Dis 1993; 48: 445-446.

6. Stewart BN, Hood CI, Block AJ. Long-term results of continuous oxygen therapy at sea level. Chest 1975; 68: 486-492.

7. Buyse B, Demedts M. Long-term oxygen therapy with concentrators and liquid oxygen. Acta Clin Belg 1995; 50: $149-157$.

8. Ringbaek T, Lange P, Viskum K. Compliance with LTOT and consumption of mobile oxygen. Respir Med 1999; 93: 333-337.

9. Clini E, Vitacca M, Foglio K, Simoni P, Ambrosino $\mathrm{N}$. Long-term home care programmes may reduce hospital admissions in COPD with chronic hypercapnia. Eur Respir J 1996; 9: 1605-1610.

10. Farrero E, Escarrabill J, Prats E, Maderal M, Manresa F. Impact of a hospital-based home-care program on the management of COPD patients receiving long-term oxygen therapy. Chest 2001; 119: 364-369.

11. Ström K. The Swedish Society of Chest Medicine. Survival of patients with chronic obstructive pulmonary disease receiving long-term domiciliary oxygen therapy. Am Rev Respir Dis 1993; 147: 585-591.
12. Silverman BG, Gross TP, Babish JD. Home oxygen therapy in medicare beneficiaries, 1991 and 1992. Chest 1997; 112: 380-386.

13. Weitzenblum E, Sautegeau A, Ehrhart M, Mammosser M, Pelletier A. Long-term oxygen therapy can reverse the progression of pulmonary hypertension in patients with chronic obstructive pulmonary disease. Am Rev Respir Dis 1985; 131: 493-498.

14. Zielinski J, Tobiasz M, Hawrylkiewicz I, Sliwinski P, Palasiewicz G. Effect of long-term oxygen therapy on pulmonary hemodynamics in COPD patients: a 6-year prospective study. Chest 1998; 113: 65-70.

15. Woodcock AA, Gross ER, Geddes DM. Oxygen relieves breathlessness in "pink puffers". Lancet 1981; 1: 907-909.

16. Gravil JH, Al-Rawas OA, Cotton MM, Flanigan U, Irwin A, Stevenson RD. Home treatment of exacerbations of chronic obstructive pulmonary disease by an acute respiratory assessment service. Lancet 1998; 351: 1853-1855.

17. Garrod R, Paul EA, Wedzicha JA. Supplemental oxygen during pulmonary rehabilitation in patients with COPD with exercise hypoxaemia. Thorax 2000; 55: 539-543.

18. Roberts CM, Bell J, Wedzicha JA. Comparison of the efficacy of a demand oxygen delivery system with continuous low flow oxygen in subjects with stable COPD and severe oxygen desaturation on walking. Thorax 1996; 51: 831-834.

19. Levi-Valensi P, Weitzenblum E, Pedinielli J-L, Racineux J-L. Three-month follow-up of arterial blood gas determinations in candidates for long-term oxygen therapy. Am Rev Respir Dis 1986; 133: 547-551. 